\title{
Les choix de vie des élèves - instituteurs issus d'une reconversion professionnelle
}

\section{Alain Domergue}

\section{(2) OpenEdition}

1 Journals

Édition électronique

URL : http://journals.openedition.org/trema/2427

DOI : $10.4000 /$ trema.2427

ISSN : 2107-0997

\section{Éditeur}

Faculté d'Éducation de l'université de Montpellier

\section{Édition imprimée}

Date de publication : 1 mai 1992

Pagination : 31-37

ISSN : 1167-315X

\section{Référence électronique}

Alain Domergue, "Les choix de vie des élèves - instituteurs issus d'une reconversion professionnelle », Tréma [En ligne], 1 | 1992, mis en ligne le 02 décembre 2013, consulté le 30 avril 2019. URL : http:// journals.openedition.org/trema/2427 ; DOI : 10.4000/trema.2427

Ce document a été généré automatiquement le 30 avril 2019

Trema 


\title{
Les choix de vie des élèves - instituteurs issus d'une reconversion professionnelle
}

\author{
Alain Domergue
}

1 La décision d'élever à la Licence le niveau de recrutement des instituteurs et de confier à l'Université la formation de l'ensemble des enseignants des premier et second degrés dans le cadre des Instituts Universitaires de Formation des Maîtres récemment créés, constitue une étape décisive de la dynamique qui a vu, à partir de la Troisième République, le niveau de recrutement des instituteurs s'élever du B.E.P.C. au D.E.U.G. en passant par le Baccalauréat alors que, parallèlement, l'institution de formation originelle tentait des approches en direction de l'enseignement supérieur en dispensant, durant quelques années, la préparation à un D.E.U.G. dit « premier degré » intégrée au cursus de formation professionnelle initiale des élèves-instituteurs de l'Ecole Normale.

2 L'une des conséquences immédiatement perceptibles de ce mouvement, renforcée dans la région Languedoc-Roussillon par la grave crise économique génératrice d'un taux de chômage particulièrement fort, a été le sensible relèvement de l'âge moyen des élèvesinstituteurs. Sont notamment apparus dans les Ecoles Normales des élèves-instituteurs adultes, certains d'entre eux ayant exercé un métier auparavant, et titulaires de diplômes et titres de capacité de haut niveau, susceptibles, aurait-il semblé, de faciliter l'obtention d'un emploi.

De l'ensemble des interrogations suscitées par cette réalité nouvelle, il a semblé intéressant d'extraire celle de la motivation et d'en faire la problématique d'une recherche consacrée aux élèves-instituteurs issus d'une reconversion professionnelle.

C'est la question de la valeur attribuée aux différents temps de la vie active et de leurs relations qui s'est trouvée au cœur de de l'hypothèse de recherche. Les définitions des différents temps de la vie active sont celles données par J. Dumazedier (1977) - Temps contraint : temps de travail professionnel et temps de travail domestico-familial. Temps libre : temps de loisirs et temps des engagements sociaux, politiques et religieux. Temps 
extra-professionnel ; temps de travail domestico-familial et temps libre, par opposition avec le temps de travail professionnel.

5 L'hypothèse de recherche adoptée pose que les personnes détentrices d'un emploi rémunéré qui décident de le quitter pour le métier d'instituteur font un choix de vie orienté vers la recherche d'une vie intégrée dans laquelle sont privilégiées des valeurs de bien-être susceptibles d'offrir une qualité d'existence, valeurs qui imprègnent la pratique professionnelle aussi bien que le temps extra-professionnel.

6 La recherche a pour objet les élèves-instituteurs issus d'une reconversion professionnelle.

7 Elle définit une population, celle des personnes qui exerçaient, effectivement, une profession rémunérée au jour où elles ont présenté les épreuves du Concours de Recrutement des élèves-instituteurs auquel elles ont par la suite été admises. Sont ainsi exclues celles qui poursuivaient des études, venaient de les terminer et n'avaient pas encore obtenu un emploi, effectuaient leur Service National, étaient sans profession et enfin se trouvaient au chômage.

Elle définit également une localisation. Le projet initial visait uniquement l'Ecole Normale du Gard à Nîmes. Toutefois, des contraintes matérielles, temporelles et organisationnelles, ont entrainé une légère modification de ce paramètre conduisant à élargir l'enquête à l'Ecole Normale de l'Hérault à Montpellier.

Elle définit ensuite une position administrative, celle d'élève-instituteur en formation à l'Ecole Normale et, pour quelques-uns, recrutés sur la liste complémentaire au concours et affectés sur un poste d'instituteur.

Du point de vue méthodologique, différentes contraintes ont imposé le choix du questionnaire.

11 Présenté sous la forme de huit pages dactylographiées, précédé de son plan, il se subdivise en trois volets et plusieurs sous-parties : origine socio-professionnelle (statut social, statut professionnel), circonstances du choix (Information, Influences, Motivation) et représentation professionnelle (La profession d'instituteur, La carrière professionnelle, La formation).

12 La majeure partie des questions sont des questions fermées, toutefois un certain nombre de questions ouvertes ont nécessité une analyse de contenu.

13 L'échantillon de la population étudiée n'a pas prétention à être représentatif de cette population dans son ensemble. Composé d'un nombre modeste de sujets, diminué des réponses inexploitables, il n'a pas paru pertinent de recourir dans son étude à des calculs de ratio qui n'auraient pas revêtu de valeur significative dans ces conditions. On a voulu mettre à jour des tendances et des ordres de grandeur qui, dans un certain nombre de cas, seront comparés à des études statistiques validées de portée nationale.

Il convient également de préciser que la première ébauche du questionnaire a été soumise à la critique de quelques instituteurs en poste depuis environ cinq ans et qui, eux-mêmes, ont connu l'expérience de vie étudiée. Leurs observations une fois intégrées, le questionnaire a été testé sur un petit groupe d'instituteurs membres d'un mouvement pédagogique ayant parcouru cette même trajectoire. La rédaction définitive s'en est suivie après la critique amicale et constructive d'un professeur d'Ecole Normale.

Les questionnaires ont été distribués accompagnés d'une explication fournie aux personnes intéressées par des professeurs et animateurs des Ecoles Normales et d'une 
lettre de présentation à l'intention des élèves-instituteurs lorsqu'ils se trouvaient sur le terrain.

La réponse se fondait sur une démarche de volontariat et la confidentialité était assurée par la remise des réponses sous enveloppe anonyme et close. L'ensemble des questionnaires remplis a été collecté par les mêmes personnes qui les avaient distribués.

L'origine socio-professionnelle du public étudié a été examinée au travers de son statut social et de son statut professionnel.

D'origine familiale plutôt modeste, le sujet-type de l'enquête est une femme mariée, âgée de 32 ans - donc plus âgée que ses camarades de promotion - ayant effectué des études à caractère scientifique ou technique qui l'ont conduite à obtenir, après un baccalauréat de type scientifique, un diplôme de niveau Bac. +2 .

Le métier antérieur offrait de réels intérêts et attraits situés essentiellement dans le registre qualitatif : relations humaines, espaces d'autonomie et intérêt intrinsèque au plan de la professionnalité.

Parallèlement, il comportait des inconvénients tenant à la médiocrité de la qualité de vie qu'il offrait, tant durant le temps de travail professionnel que durant le temps extraprofessionnel, aux difficultés dans les relations humaines (citées précédemment comme source d'intérêt) et aux pesanteurs organisationnelles et structurelles.

21 Au bilan, le métier antérieur était perçu comme un métier intéressant, comportant certes des inconvénients mais ne faisant pas massivement l'objet d'une réaction de rejet de la part de personnes qui accordent une importance primordiale aux relations humaines, à la dimension de réalisation personnelle et à l'existence d'espaces de liberté.

Les circonstances du choix ont été examinées à partir des variables informations, influences subies et motivation énoncée.

Au moment où avait lieu l'enquête, les élèves-instituteurs interrogés estimaient avoir été informés de manière suffisante ou complète. Leur choix de devenir instituteur est l'aboutissement d'une démarche personnelle, cohérente avec leur profil sociologique. Ils perçoivent ce métier comme socialement utile et l'ont choisi par intérêt pour les enfants, et aussi parce qu'il offre l'opportunité d'une meilleure qualité de vie professionnelle comme extra-professionnelle.

24 Les représentations de la profession, de la carrière professionnelle et de la formation permettent de tracer le tableau de la représentation professionnelle du métier d'instituteur.

25 Les personnes interrogées s'accordent sur le caractère d'utilité sociale de la profession d'instituteur qu'elles viennent d'embrasser. A ce titre, certainement, elles estiment que ce métier est considéré. Ce point de vue est en harmonie avec l'appréciation précédente mais en décalage par rapport à l'opinion publique lorsqu'elle s'exprime à ce sujet dans l'enquête publiée dans les numéros 1360 et 1361 de l'hebdomadaire Le Nouvel Observateur (1990) ${ }^{1}$.

26 Elles jugent également que ce métier, placé au service d'autrui, est insuffisamment rémunéré sans que pour autant cette dimension soit perçue comme éminente et fasse l'objet d'une critique radicale.

Cette sérénité manifeste d'appréciation se heurte cependant au sentiment qu'éprouvent ces personnes selon lequel leurs pairs ne sont pas à la hauteur de leurs responsabilités. 

s'intègre dans une vision dynamique qui en appelle à la nécessité d'un apprentissage de savoir-faire (la nature du questionnaire ne permettant pas de se prononcer sur la dimension des savoirs) et qui souligne l'importance de la maturation du sujet. Elle se réfère, en second lieu, à l'existence d'un terrain personnel prédisposé pour cette profession et aussi à des aspects de son exercice relevant du domaine de l'intuition, de la sensibilité.

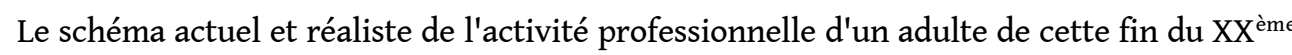
siècle est en partie intégré. Il se positionne en rupture avec le modèle en voie de disparition du métier unique exercé durant toute la vie active.

Il conduit les sujets observés à envisager la possibilité d'une nouvelle reconversion dans leur carrière professionnelle ou, tout au moins, de bénéficier de formules de gestion souple de ce cursus, dans le but, une nouvelle fois, de rassembler les conditions propres à préserver une appréciable qualité de vie.

31 Quant au modèle de formation alors en vigueur, celui des Ecoles Normales, il est perçu comme pertinent puisque capable de préparer au métier d'instituteur selon des orientations ouvertes et actives. Les modifications attendues en la matière sont source d'inquiétude pour ceux qui s'estiment en mesure de se prononcer.

32 La représentation professionnelle (carrière, profession, formation) est traversée par quelques grands traits : perception dynamique, intégration de la maturité dans les choix et options conçues comme évolutives, attitude d'ouverture sereine devant ces caractéristiques, confiance dans l'instrument de formation.

\section{La motivation}

33 L'interprétation des résultats impliquait d'adopter un modèle conceptuel de la motivation opérant et utile sur le terrain d'étude. Après qu'ait été écarté le modèle innéiste des niveaux hiérarchiques de besoins proposé par Maslow (1954) le choix s'est porté sur une construction théorique qui se fonde sur l'analyse du processus de motivations, mécanisme complexe qui met en liaison travail, culture, échelle des valeurs personnelles, personnalité, information accessible et capacité à la traiter, etc.

Le modèle employé, dit modèle V.I.E., introduit la dimension subjective dans la dynamique motivationnelle. Il s'appuie sur trois concepts : Valence (V), Instrumentalité (I) et Expectation (E) qui sont autant de variables intermédiaires observables, compréhensibles, pouvant être déterminées et modifiées par le jeu des évolutions sociales et personnelles.

35 L'Expectation s'intéresse à la cohérence entre la tâche et les qualités et compétences que l'on se reconnaît. Elle estime ce que l'on se croit capable de faire à partir du rapport que l'on établit entre l'opinion que l'on a de soi (les qualités et les insuffisances que l'on s'attribue, se reconnaît) et les efforts que l'on est prêt à fournir.

36 Le public de l'enquête possède une représentation de soi ("self-concept») positive. Son entrée dans la vie professionnelle s'est déroulée sous le signe de la réussite : succès dans des études scientifiques réputées difficiles et sélectives, cursus universitaire, insertion socio-professionnelle réussie dans une région fortement frappée par le chômage et 
utilisant principalement une main d'œuvre peu qualifiée, niveau de vie satisfaisant, métier intéressant malgré tout.

En regard, l'effort à fournir n'est pas hors de portée. Ce public est détenteur d'un emploi, ses revenus sont corrects et il est entouré familialement. Il tire de cette position une certaine assurance, source de cette tranquillité d'esprit qui le place en situation favorable (par exemple dans les examens et concours, ainsi que le montraient P. Bourdieu et J.C. Passeron, 1964). Naturellement, sa maturité, son expérience de la vie, l'ont préparé, à ses propres yeux, à se présenter au concours. La représentation qu'il a du métier d'instituteur et de sa formation professionnelle l'assurent de sa capacité à s'y confronter, d'autant qu'il est prêt à de nouvelles réorientations dans son nouveau métier, limitées il est vrai. De plus, il choisit l'Ecole Normale du département dans lequel il est installé, en ayant l'assurance d'être nommé dans ce département à la fin de sa formation. Le risque d'un échec peut être assumé, il n'entamera pas le «self-concept » déjà constitué qui, confusément peut-être, conduit à imaginer une suite de carrière favorable et équilibrée.

Ce public s'est senti en mesure de réussir sa reconversion professionnelle et d'assumer son nouveau métier.

L'Instrumentalité exprime la manière dont est perçue la relation entre le travail fourni et ce que rapporte le travail. Pour qu'un travail soit motivant, il doit être un moyen efficace d'obtenir un certain résultat recherché.

En l'occurrence, le travail c'est le métier et la carrière d'instituteur non pas tels qu'ils sont vécus et donc appréciés mais bien tels qu'ils sont figurés dans les représentations que le public de l'enquête s'en fait, et aussi à partir, il est vrai, d'informations objectives dont il dispose.

41 Le résultat recherché se résume à deux exigences : rassembler les conditions d'une vie qualitativement avantagée dans le temps de travail et en dehors, et être professionnellement au contact d'enfants.

42 La première condition est réunie pour ce public: horaires (en masse et distribution), congés, affectation dans le département, relations humaines, indépendance et autonomie dans le métier, etc. La deuxième condition résulte de la mission d'un métier tourné vers les enfants.

Il n'est donc pas besoin de développer plus longuement pour reconnaître que cette dimension subjective joue pleinement, qui conduit les sujets à percevoir la liaison étroite existant entre le travail et les avantages recherchés qu'il compte en tirer.

Est naturellement présente l'idée que les résultats et conditions recherchés dans le métier d'instituteur sont affectés d'une valeur réelle.

Cette valeur est la Valence que le sujet attribue aux récompenses recherchées offertes par le métier. Il faut souligner fortement le caractère subjectif et personnel de ce troisième concept qui attribue des attraits au travail en fonction d'une opinion personnelle.

Ainsi, si l'on considère les revenus, ils ne seront pas intégrés dans la dimension valence dans la mesure où, bien que jugés assez satisfaisants par les sujets de l'étude, ils ne pèsent pas plus en tant que motif du choix de la nouvelle profession que ce qu'ils étaient pris en compte dans l'appréciation portée sur l'ancien métier.

Lorsque les sujets de l'enquête s'exprimaient sur leur ancien métier en terme d'avantages et d'inconvénients, ils s'arrêtaient sur un certain nombre de caractéristiques considérées 
comme essentielles. Il est intéressant d'observer qu'ils se situent également sur ces mêmes caractéristiques lorsqu'ils s'expriment, du même point de vue, sur la profession d'instituteur. Alors, ils les affectent d'une valeur positive, leur attribuant de la sorte le statut d'attraits, garants de la qualité de vie. Ce sont ces caractères-là qui ont été recensés au cours de l'analyse conduite précédemment selon l'axe de l'instrumentalité et qu'il n'est donc pas utile d'énumérer une nouvelle fois.

L'investigation conduite sur la considération provoque chez les élèves-instituteurs interrogés une réponse relativement neutre, non susceptible de motiver leur choix. Elle n'altère pas plus une image de soi raisonnablement optimiste déjà observée. Dans la mesure où elle ne serait pas amenuisée par la suite, elle pourrait être susceptible de faire contrepoids au sentiment de malaise qui pourrait résulter des aspirations énoncées d'accès aux valeurs d'autonomie, de responsabilité, de risque en somme, valorisées et gratifiantes mais dans le même temps fondamentalement anxiogènes selon le sociologue Alain Ehrenberg (1989).

L'application du modèle motivationnel « V.I.E. » a permis de montrer que la démarche de choix du métier, telle qu'elle apparaît au dépouillement des questionnaires renseignés par les élèves-instituteurs issus d'une reconversion professionnelle, est le fait de personnes disposant d'une représentation de soi positive compatible avec l'effort nécessité par la présentation au C.R.E.I. et par le métier d'instituteur. Ensuite, ils estiment pouvoir trouver dans le travail fourni les récompenses qu'ils en attendent (qualité de vie intra et extra-professionnelle et contact avec les enfants). Enfin, ils manifestent un fort attachement à ces récompenses, aussi bien quand ils les qualifient en les rapportant à leur représentation du métier d'instituteur, qu'au moment où ils sont amenés à s'exprimer sur leur ancien métier.

En conclusion, il est donc possible de faire le constat de la compatibilité des résultats avec l'hypothèse adoptée pour la recherche.

51 Les élèves-instituteurs issus d'une reconversion professionnelle font un choix de vie où le temps professionnel est conçu comme un temps d'une vie où l'on s'applique à mieux équilibrer les valeurs qui méritent d'être cultivées dans la recherche du bien-être.

Ces valeurs doivent être présentes dans le métier : autonomie, responsabilité, temps pour soi et pour son environnement socio-culturel : du qualitatif plutôt que du quantitatif en un mot.

53 Toutefois, l'hypothèse a été définie de manière trop restrictive. L'étude montre en effet que, se centrant exclusivement sur les personnes étudiées, l'hypothèse a négligé de considérer explicitement l'objet de la mission des instituteurs, c'est-à-dire les enfants. En revanche, les réponses aux questionnaires donnent à cet élément un véritable statut de motif de choix de ce métier, sans rapport toutefois avec le registre de la vocation, venant même faire équilibre aux raisons tenant à la recherche de la qualité de vie.

Dans cette étude consacrée à la motivation d'un choix professionnel, la discrétion du propos sur la professionnalité dans le métier d'instituteur est en décalage avec l'intérêt qu'y portent certaines études plaidant pour une transformation de la profession enseignante reposant sur la professionnalisation. Cette dimension semblerait pourtant susceptible de mettre en meilleure adéquation cette profession avec les finalités d'aujourd'hui et aussi de porter remède au malaise professionnel des enseignants dû à une dégradation de leur identité professionnelle et de leur image sociale. 
arque suggère une perspective de réflexions et de recherches. Au moment où la question de la formation des enseignants s'appréhende selon une dimension européenne et où notre pays la porte au niveau universitaire, le paramètre de la professionnalisation du métier d'enseignant gagnerait à faire l'objet d'une attention particulière. De la sorte seraient susceptibles d'émerger des enseignements propres à amener cette profession à une plus grande efficacité et, dans le même temps, à rendre à ses membres une image de soi positive et le meilleur confort d'existence auquel ils aspirent.

\section{BIBLIOGRAPHIE}

BERGER Ida, Les instituteurs d'une génération à l'autre, P.U.F. 1979.

BOURDIEU Pierre et DARBEL. Alain, L'amour de l'art, MINUIT 1969.

BOURDIEU Pierre et PASSERON Jean- Claude, Les héritiers, MINUIT 1964.

DESBROUSSES Hélène, Instituteurs et professeurs, Matériaux pour l'analyse d'un groupe social, EDIRES, 1982.

DUMAZEDIER Joffre, Vers une civilisation du loisir ?, SEUIL 1962.

DUMAZEDIER Joffre. Sociologie empirique du loisir, SEUIL 1977.

EHRENBERG Alain, L'individu sous perfusion. Société concurrentielle et anxiété de masse. Esprit, JuilletAoût, 1989.

ESTEVE JOSÉ. M. et FRACCHIA Alice F., Le malaise des enseignants, Revue française de pédagogie, $n^{\circ} 84$, 1988.

HUBERMAN Michael. Les phases de la carrière enseignante : essai de description et de prévision, Revue Française de pédagogie, $n^{\circ} 8,1989$.

LEVY-BOYER Claude, La crise des motivations, P.U.F., 1984.

LOUVET Andrée, Recherche et formation, $n^{\circ}$ 6, 1989.

MASLOW A.H., Motivation and personnality, New York, HARPER, 1954.

MINISTERE DE L'ÉDUCATION NATIONALE, Tableaux statistiques 1988-1989. Recensement des personnels du corps des instituteurs des établissements des premier et second degrés. Situation au 31 décembre 1988. Avril 1990.

MINISTERE DE L'ÉDUCATION NATIONALE, Tableaux statistiques 1987-1988. Les instituteurs en formation dans les Ecoles Normales. Récapitulation nationale. Mai 1989.

MUCCHIELLI Alex, Les motivations. Que sais-je ? P.U.F., 1981.

PORCHER Louis, Education esthétique et formation des instituteurs, E.S.F., 1975. 


\section{NOTES}

1. Les items du questionnaire relatifs aux notions d'utilité sociale, de conscience professionnelle, de considération sociale et de revenus sont repris des items du sondage SOFRES pour cet hebdomadaire, représentatif de l'ensemble de la population âgée de 18 ans et plus. Il a donc été possible de disposer d'une référence statistique nationale.

\section{RÉSUMÉS}

Des personnes titulaires d'un emploi rémunéré rompent avec leur ancien métier pour choisir celui d'instituteur. Quelle est leur motivation? L'application d'un modèle dynamique de la motivation aux résultats obtenus par un questionnaire portant sur l'origine socio professionnelle, les circonstances de la décision et la représentation de la profession, met en évidence un choix de qualité de vie.

Individuals with a remunerated occupation resign their position and choose to become primary teachers. What motivates them to do this? Applying a dynamic model of motivation to answer to the questionnaire on the social- professional origins as well as the circumstances in which the decision was made and the image of the profession, it becomes obvious that their choice is guided by a search for a better quality of life.

\section{INDEX}

Mots-clés : Choix de vie, élèves-instituteurs, reconversion professionnelle

\section{AUTEUR}

\section{ALAIN DOMERGUE}

Inspection départementale AIS de Nîmes 